\title{
PAPER
}

Check for updates

Cite this: J. Mater. Chem. A, 2018, 6 , 1485

Received 13th November 2017

Accepted 22nd December 2017

DOI: $10.1039 / \mathrm{cta10002g}$

rsc.li/materials-a

\section{Synthesis of STW zeolites using imidazolium-based dications of varying length $\dagger$}

\begin{abstract}
Peng Lu, ${ }^{\text {abc }}$ Luis Gómez-Hortigüela, (D) d Lei Xuª and Miguel A. Camblor (iD) *c
Dications consisting of 1,2-dimethylimidazolium units linked by chains of 4, 5 or 6 methylene groups display structure direction towards the chiral zeolite STW as racemic conglomerates with decreasing strength as the length of the linker increases. A phase transformation from the denser and less strained MTW to the less dense and more strained STW reveals significant stabilization in the case of the 4-methylene dication, which is able to produce STW even in the absence of germanium (i.e., for a pure silica composition), and to a lesser extent for the 5-methylene derivative. When Ge is introduced in the synthesis the crystallization is easier and the crystallization field is wider, with Ge preferentially occupying positions at the double four ring units of the STW framework, as revealed by Rietveld refinement from synchrotron data. Quantum mechanics calculations show that the interaction energy diminishes as the length of the linker increases, in agreement with the experimental findings. In addition, calculation of the ${ }^{13} \mathrm{C}$ NMR chemical shieldings and comparison with experimental spectra allowed us to identify different conformations and orientations of the imidazolium rings in the asymmetric STW cavities, depending on the spacer length. An exploratory study strongly suggests that an asymmetric derivative with additional methyl groups at both sides of the tetramethylene chain could afford the crystallization of the homochiral zeolite even as a pure silica material.
\end{abstract}

\section{Introduction}

Chiral zeolites in enantiomerically pure form might be of enormous interest for adsorption/separation and catalysis applications requiring chiral recognition, such as, for instance, resolution of racemic mixtures or enantioselective catalysis. Hence, the synthesis of homochiral zeolites has been one of the most attractive goals in the field ever since, at least, the discovery of the chiral nature of polymorph A of zeolite beta. ${ }^{1}$ For almost three decades, however, very little success was

${ }^{a}$ Dalian National Laboratory for Clean Energy, Dalian Institute of Chemical Physics, Chinese Academy of Sciences, Dalian 116023, P. R. China

${ }^{b}$ University of Chinese Academy of Sciences, Beijing 100049, P. R. China

'Instituto de Ciencia de Materiales de Madrid, Consejo Superior de Investigaciones Científicas (ICMM-CSIC), c/ Sor Juana Inés de la Cruz 3, 28049 Madrid, Spain. E-mail: macamblor@icmm.csic.es

${ }^{d}$ Instituto de Catálisis y Petroleoquimica, Consejo Superior de Investigaciones Cientificas (ICP-CSIC), c/ Marie Curie 2, 28049 Madrid, Spain

$\dagger$ Electronic supplementary information (ESI) available: ${ }^{1} \mathrm{H}$ and ${ }^{13} \mathrm{C}$ NMR in $\mathrm{D}_{2} \mathrm{O}$ of the three organic dications used in this work, tabulated results for the synthesis of pure silica and germanosilicate zeolites, XRD patterns of additional phases, ${ }^{29} \mathrm{Si}$ and ${ }^{13} \mathrm{C}$ CPMAS NMR spectra of as-made STW zeolites, thermogravimetric and differential thermal analysis in flowing air of as-made STW zeolites, tables and Rietveld plot for as-made Ge,Si-4BDMI-STW, molecular mechanics location of 4BDMI in $P 6_{1} 22$ and $P 6_{5} 22$ enantiomorphs, additional figures of the STW cavities and of different locations of the dications and radial distribution function of $\mathrm{C}(\mathrm{e})$ of 4BDMI in different configurations. CCDC 1578113. For ESI and crystallographic data in CIF or other electronic format see DOI: $10.1039 / \mathrm{c} 7 \mathrm{ta} 10002 \mathrm{~g}$ achieved until very recently, when Brand et al. demonstrated the synthesis of enantiomerically enriched germanosilicate and aluminogermanosilicate zeolites with the chiral STW structure, containing a helicoidal channel, and their use in chiral recognition in adsorption and catalysis processes. ${ }^{2}$ The synthesis made use of an imidazolium-based dication as an organic structure-directing agent (SDA), in which tetramethylated imidazolium moieties were connected through a chiral linker. Since the imidazolium rings need to be sited in adjacent cages of the helicoidal channel, the asymmetric nature of the linker determined to a certain extent the handedness of the pores and hence that of each microcrystal. By using enantiomerically pure dications, the crystallization produced not pure but apparently largely enriched solids (scalemic conglomerates) rather than the racemic conglomerate expected to crystallize with a symmetric SDA.

During our ongoing project on the structure-directing ability of imidazolium-based cations in the synthesis of zeolites, we have found that dimethylated imidazolium-based dications with linkers of 4, 5 and 6 methylenes were also able to produce STW zeolites. Interestingly, even pure silica zeolites, with a much higher thermal and hydrothermal stability than germanium based materials, can be synthesized. Our further work was driven also by the idea that the imidazolium moieties of the dications should be located in adjacent cages of the STW structure and that, hence, this could open a way to control the crystallization of enantiopure solids. Thus, we have explored 
computationally the possibility of imposing the appropriate spatial arrangement of the imidazolium rings and then of the resulting cages to produce enantiopure STW zeolites. Here we report our experimental and theoretical study on the synthesis of STW zeolites using imidazolium dications as well as our computational exploration of several asymmetric derivatives of the best SDA found.

\section{Experimental section}

\section{Synthesis of the organic cations}

The organic cations used in this work are doubly charged cations consisting of two 1,2-dimethylimidazolium moieties connected by a linear methylene chain with $n=4,5$ and 6 carbon atoms (Fig. 1). These dications, denoted as $n$ BDMI, were synthesized by reacting 1,2-dimethylimidazole with the corresponding linear $\alpha, \omega$-dibromoalkane. In a typical synthesis procedure, $0.14 \mathrm{~mol}$ (13.733 g) of 1,2-dimethylimidazole (SigmaAldrich, 98\%) was dissolved in $50 \mathrm{ml}$ of chloroform (SigmaAldrich, 99\%) for 4BDMI and 6BDMI or methanol (Aldrich, 99.8\%) for 5BDMI. The linear alkyl bromide (0.07 mol, SigmaAldrich, 98\%, $n=4-6$ ) was then added, followed by another $50 \mathrm{ml}$ of the corresponding solvents. The solution was kept under magnetic stirring at room temperature for 4-7 days. Solid bromide salts of 4BDMI and 6BDMI with high purity were obtained after filtration and rotoevaporation. For 5BDMI, a purification process was adopted by dissolving the product in $20 \mathrm{ml}$ of methanol (Aldrich, 99.8\%) and the pure bromide salts were recrystallized by adding $60 \mathrm{ml}$ of ethyl acetate (Sigma-Aldrich, 99\%), and were then filtered and dried.

The nature and purity of the SDAs were verified by ${ }^{1} \mathrm{H}$ and ${ }^{13} \mathrm{C}$ NMR in $\mathrm{D}_{2} \mathrm{O}$ (Fig. $\mathrm{S} 1$ and $\mathrm{S} 2, \uparrow$ respectively). The dibromide salts were converted to their corresponding hydroxide forms by anion exchange using Dowex anion exchange resin 550A (OH) (Sigma-Aldrich, 1.1 mequiv./1 ml). The hydroxide solution was concentrated by rotoevaporation under vacuum. The concentration of the final solution was determined by titration with $\mathrm{HCl} 0.1 \mathrm{~N}$ using phenolphthalein as an indicator.

\section{Synthesis of zeolites}

For the pure silica zeolite synthesis, the starting gel was prepared as follows. Tetraethylorthosilicate (TEOS, 98\%, SigmaAldrich) was hydrolyzed under magnetic stirring at ambient temperature in the alkaline aqueous solution of the organic cations. Some water and all ethanol, produced by the hydrolysis of TEOS, were evaporated, which was monitored by weight loss.

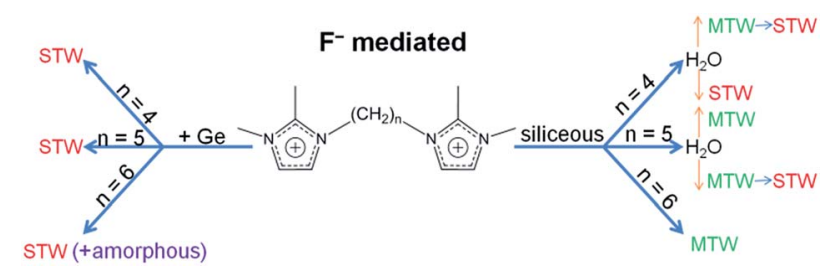

Fig. 1 Organic cations used in this work as SDAs with a scheme of their structure-directing ability.
The stirring was stopped when the desired $\mathrm{H}_{2} \mathrm{O} / \mathrm{SiO}_{2}$ ratio was achieved. Then, the required amount of $\mathrm{HF}$ aqueous solution (Sigma-Aldrich, 48\%) was added and the mixture was homogenized with a spatula by hand for $15 \mathrm{~min}$. The final gel composition was $\mathrm{SiO}_{2}: 0.25 \mathrm{R}(\mathrm{OH})_{2}: 0.5 \mathrm{HF}: x \mathrm{H}_{2} \mathrm{O}$, where $x$ varies between 3 and 10 and $\mathrm{R}$ stands for the different SDAs used.

Gels for germanosilicate synthesis started with the addition of germanium dioxide (Aldrich, 99.998\%) to the aqueous solution of SDAs, and then, TEOS (Sigma-Aldrich, 98\%) as a silica source was added followed by the same procedure described above. The final starting gel composition was $(1-x) \mathrm{SiO}_{2}: x \mathrm{GeO}_{2}: 0.25 \mathrm{R}(\mathrm{OH})_{2}$ : $0.5 \mathrm{HF}: y \mathrm{H}_{2} \mathrm{O}$, where $0 \leqq x \leqq 0.5$, and $y$ was $3,5,10$ and 15 .

The gels were distributed into Teflon lined stainless steel autoclaves which were heated in an oven at different temperatures while tumbling (about $60 \mathrm{rpm}$ ). The autoclaves were taken out at different time intervals and quenched with tap water. The resulting solids were thoroughly washed with deionized water, filtered and dried at $100{ }^{\circ} \mathrm{C}$.

\section{Characterization}

Powder X-ray diffraction (PXRD) was used to identify the recovered solids. The diffraction patterns were obtained on a Bruker D8 Advance diffractometer using $\mathrm{Cu} \mathrm{K} \alpha$ radiation $(\lambda=$ $1.5418 \AA$ A). Synchrotron X-ray powder diffraction data of the silicogermanate sample synthesized with 4BDMI were also collected in capillary mode $(0.8 \mathrm{~mm}$ internal diameter $)$ at the Spanish beamline BM25A in the ESRF, Grenoble $(\lambda=0.56383$ A). Field emission scanning electron micrographs were collected using a Philips XL30 S-FEG. Multinuclear magic angle spinning (MAS) NMR spectroscopy of the as-synthesized samples was performed at room temperature on Bruker AV400-WB equipment and the details have been given elsewhere. ${ }^{3}$ The amounts of organic moieties occluded in the asmade zeolites were determined by $\mathrm{CHN}$ elemental analysis using a LECO CHNS-932 analyzer. Thermal gravimetric analysis was carried out on a SDT Q600 TA instrument under air flow $\left(100 \mathrm{ml} \mathrm{min}^{-1}\right.$ ) heating from $25{ }^{\circ} \mathrm{C}$ to $1000{ }^{\circ} \mathrm{C}$ (with a heating rate of $\left.10{ }^{\circ} \mathrm{C} \mathrm{min}^{-1}\right)$.

\section{Computational details}

The computational methodology employed to study the structure directing effect of the bis-imidazolium dications during the crystallization of the STW zeolite was based on a combination of molecular mechanics and quantum mechanics simulations, as implemented in Materials Studio. ${ }^{4}$ The geometry of the zeolite structure $\left(P 6_{1} 22\right.$ space group) (including the fluoride anions) was taken from our previously reported work; ${ }^{5}(1 \times 1 \times 1)$ STW primary unit cells $\left(\mathrm{Si}_{60} \mathrm{O}_{120}\right)$ were used (under periodic boundary conditions) since these were large enough to prevent interactions between image cells.

The location and the interaction energy of the different SDA cations with the framework were determined through a two-step procedure: (i) first a search for the most stable location of the SDA cations in different configurations through molecular mechanics (simulated annealing) calculations, followed by (ii) 
a DFT+D study of these optimized systems (including calculation of the ${ }^{13} \mathrm{C}$ NMR shielding).

For the initial molecular mechanics calculations, the molecular structures of the cations and the interaction energies with the framework are described with the cvff forcefield; for the sake of comparison, calculations were also performed with the Dreiding forcefield; during these molecular mechanics calculations, the atomic coordinates of the framework atoms were kept fixed. The atomic charge-distribution of the dications was obtained from DFT + D calculations, using the B3LYP hybrid functional and the ESP charge calculation method, setting the total net charge to +2 . The SDA cations were initially docked in the STW framework through Monte-Carlo simulations, using the sorption module in Materials Studio. ${ }^{6}$ The most stable location for the organic cations was obtained by means of simulated annealing calculations. The asymmetric nature of the imidazolium rings of the dications used in this work implies that there are two possible orientations of each imidazolium ring of the dications within the STW cavities: with the central methyl group in the imidazolium ring (marked $\mathrm{C}(\mathrm{e})$ in Fig. 2) pointing towards a $10 R$ - or an $8 R$-window (hereafter, these orientations will be referred to as $10 R$ and $8 R$ configurations); since there are two imidazolium rings per molecule, this gives rise to 3 different configurations for each dication, with both rings in the $10 R$ configuration $(10 R 10 R)$, both in the $8 R$ configuration $(8 R 8 R)$, or one on each $(10 R 8 R)$. Due to the confinement provided by the STW cavities, the simulated annealing procedure did not enable free rotation of the imidazolium rings around the $\mathrm{N}-\mathrm{CH}_{2}$ axis to explore the conformational space, even despite the high temperature of the simulation $(700 \mathrm{~K})$. Hence, the three possible configurations were manually built and studied, and the most stable location was determined by simulated annealing. This first set of calculations allowed us to find the most stable location for the SDA dications in the different configurations.

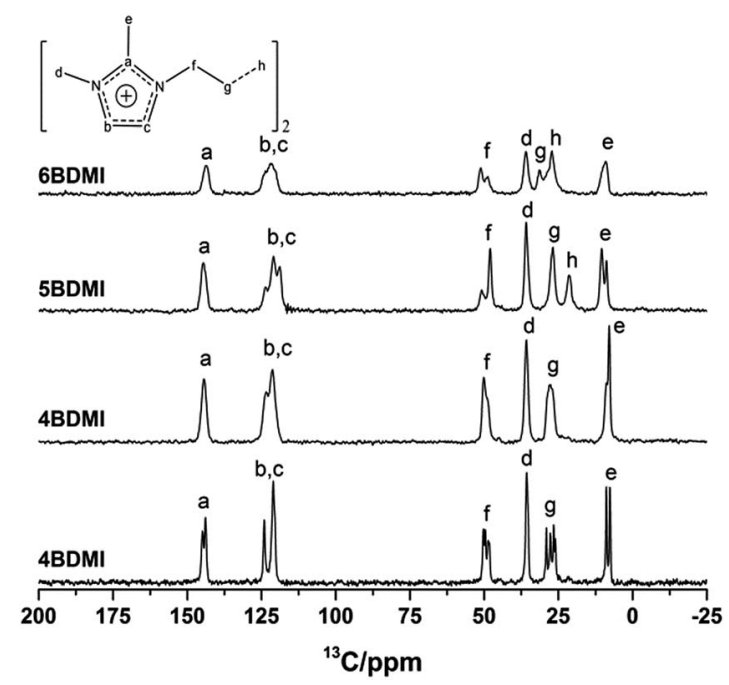

Fig. $2{ }^{13} \mathrm{C}\left\{{ }^{1} \mathrm{H}\right\}$ CPMAS NMR spectra of as-made (germano)silicate STW zeolites (from bottom to top): 4BDMI-Si-STW, 4BDMI-Si,Ge-STW $\left(\mathrm{Ge}_{\mathrm{f}}=0.5\right), 5 \mathrm{BDMI}-\mathrm{Si}, \mathrm{Ge}-\mathrm{STW}\left(\mathrm{Ge}_{\mathrm{f}}=0.1\right)$ and 6BDMI-Si, Ge-STW $\left(\mathrm{Ge}_{\mathrm{f}}\right.$ $=0.5)$.
These systems were then studied by DFT+D (CASTEP). $\mathrm{DFT}+\mathrm{D}$ geometry optimisations were performed using planewaves as a basis set (with a cut-off of $489.8 \mathrm{eV}$ ), and the PBE generalized gradient approximation as a functional, ${ }^{7}$ as implemented in the CASTEP module in Materials Studio; ${ }^{8}$ in this case, the coordinates of all atoms were relaxed. We then obtained the final interaction energies by subtracting the energy of the isolated zeolite and SDA cations from the total energy of the system, and related these to the structure-directing efficiency of the different SDA dications. Calculation of the NMR parameters was performed with the gauge-including projector augmentedwave method (GIPAW) developed by Pickard and Mauri. ${ }^{9}$ This computational methodology has been shown to accurately predict the NMR properties of zeolite materials. ${ }^{10}$ The chemical shift for a nucleus in a given position $(\delta(r))$ is then defined as:

$$
\delta(r)=\sigma_{\text {ref }}-\sigma(r)
$$

where $\sigma(r)$ is the isotropic shielding obtained in the calculations; shieldings of each $\mathrm{C}$ in the three equivalent dications present per STW u.c. (which were very similar to each other) were averaged. In order to compare with the experimental ${ }^{13} \mathrm{C}$ chemical shifts, we chose a $\sigma_{\text {ref }}$ of $174 \mathrm{ppm}$, so that the theoretical and experimental chemical shifts roughly coincide.

The second part of the calculations involved the study of the effect of adding different alkyl groups on the $\mathrm{C} 4$ spacer of 4BDMI, thus making some of these $\mathrm{C}$ atoms stereogenic centres, and hence making the dications chiral. For these calculations we loaded one dication in the 3 different orientations previously mentioned in the STW framework, and determined the most stable configuration by simulated annealing based on the cvff and Dreiding force-field methodologies previously described. In this case, we analyzed the difference in the interaction energy of the two enantiomers of the chiral dications with the STW framework (in the $P 6_{1} 22$ space group); we term this "enantiomeric energy difference". All the energies are given in kcal $\mathrm{mol}^{-1}$ per STW u.c.

\section{Results and discussion}

\section{Summary of zeolite synthesis results}

Table $\mathrm{S} 1 \dagger$ summarizes synthesis conditions and products obtained using the three different organic cations both with pure silica and germanosilicate compositions at 175 and $150{ }^{\circ} \mathrm{C}$ and at varying $\mathrm{H}_{2} \mathrm{O} / \mathrm{SiO}_{2}$ ratios. Structure-direction is schematically shown in Fig. 1. The XRD patterns for the as-made STW zeolites are shown in Fig. S3. $\dagger$

With regard to the pure silica synthesis, two zeolite phases with different framework densities (FD) were obtained: STW (FD $\left.=16.4 \mathrm{~T} / 1000 \AA^{3}\right)$ and MTW $\left(\mathrm{FD}=18.2 \mathrm{~T} / 1000 \AA^{3}\right)$. The latter was the only crystalline product reported in a prior study in hydroxide media using the same linear SDAs. ${ }^{11}$ Contrasting with the hydroxide synthesis, the chirally pure silica HPM-1 zeolite (STW) was obtained at $175{ }^{\circ} \mathrm{C}$ at high concentration (low $\mathrm{H}_{2} \mathrm{O}$ / $\mathrm{SiO}_{2}$ ratio) using either 4BDMI or 5BDMI as SDAs, while for higher dilutions structure-direction shifted to some extent towards MTW. This constitutes a further example of 
Villaescusa's rule: lower framework density phases are favored at lower water/silica ratios. ${ }^{12}$ This empirical rule has been found generally applicable to silica zeolite synthesis via the fluoride route. As for the pure silica synthesis with 6BDMI, MTW was the only phase despite the variation of water/silica ratios and temperatures within the limited range of our synthesis trials. Since the synthesis of HPM-1 is also less favored with 5BDMI than with 4BDMI, the structure-directing effect of these dications towards STW decreases as the length of the linker increases $(4 \mathrm{BDMI}>5 \mathrm{BDMI}>6 \mathrm{BDMI})$. We note here, however, that we obtained no hint of STW zeolite for a shorter dication containing a trimethylene linker (3BDMI), which may perhaps be due to the known decreased stability of dications with charges separated by a trimethylene unit. ${ }^{\mathbf{1 3 1 4}}$

It is worth mentioning here that an in situ transformation process from the denser MTW phase to the less dense STW phase was observed with both 4BDMI and 5BDMI (Table S1 $\dagger$ ). This is, to the best of our knowledge, the third example in which a high density phase transforms in situ into a low density porous phase (firstly reported for TON to ITW, ${ }^{15}$ and then for MTW to ITW). ${ }^{16}$ The phase transformation is more favored with 4BDMI than with 5BDMI, since it occurred and was completed even at intermediate concentration with 4BDMI, while it required higher concentrations and was not completed for 5BDMI after longer crystallization times. This again points to a stronger directing effect of 4BDMI towards STW. The transformation is observed in spite of the pure silica STW being not only less dense than MTW but also highly strained due to the presence of a high density of double-4-rings (D4Rs, with $80 \%$ of tetrahedral $\mathrm{T}$ sites in such units). In other words, the pure silica MTW to STW transformation occurs despite the fact that the former is probably much more stable than the latter in the calcined form. Thus, the transformation from MTW to STW using 4BDMI and, with a weaker strength, 5BDMI, reveals a significant stabilization effect of the organic cations and $\mathrm{F}^{-}$anions occluded in the zeolite that is able to revert the stability trend of the neat (i.e., devoid of guests) frameworks. As reported earlier for other systems, the strain associated with $\mathrm{D} 4 \mathrm{R}$ units in pure silica compositions can be relaxed by host-guest interactions that polarize the $\mathrm{Si}-\mathrm{O}$ bond, thus enhancing the flexibility of the silica framework. ${ }^{15}$

When $\mathrm{GeO}_{2}$ was introduced, the STW phase dominated the crystallization field in a much wider range of conditions using all three SDAs. As more effective SDAs towards STW in the pure silica trials, 4BDMI and 5BDMI in combination with $\mathrm{GeO}_{2}$ made the synthesis range wider and the crystallization rate faster. As for 6BDMI, which structure-directed only the one-dimensional MTW phase in pure silica trials and ill-crystallized unknown phases at low Ge contents (Fig. S4†), it demonstrated some structure-directing effect towards STW only when the Ge content was high (Ge molar fraction, $\mathrm{Ge}_{\mathrm{f}}=\mathrm{Ge} / \mathrm{T}, \mathrm{T}=(\mathrm{Si}+\mathrm{Ge})$, of around 0.5 ), although minor amounts of crystalline $\mathrm{GeO}_{2}$ or some amorphous phase coexisted in the products. Early reports have shown that $\mathrm{Ge}$ is preferentially incorporated into $\mathrm{D} 4 \mathrm{R}$ cages together with $\mathrm{F}^{-}$and this apparently helps stabilizing the D4R units. ${ }^{17}$ The introduction of Ge relaxes the geometric constrains in the D4R by increasing the T-O-T bond length and decreasing the T-O-T angle, leading to more stable structures. Thus, the D4R containing zeolitic structure is believed to be favored by the introduction of Ge in combination with $\mathrm{F}^{-}$.

Prior recent reports by Davis's group have shown that STW could also be obtained with similar dications based on the tetramethyleneimidazolium moiety. With the tetramethylene linker STW crystallized in competition with CIT-7 in the absence of Ge, ${ }^{\mathbf{1 8 , 1 9}}$ while it could be obtained pure in the presence of Ge using the pentamethylene linker. ${ }^{19}$

\section{Zeolite characterization}

The chemical composition details of the as-made SDA-zeolites are listed in Table 1 . The $\mathrm{C} / \mathrm{N}$ ratio of the organics occluded in the zeolites is close to the theoretical value in each case, suggesting that the SDAs are roughly intact. For pure silica STW zeolites synthesized with 4BDMI, there are three dications in a unit cell, indicating that one SDA spans two $\left[4^{6} 5^{8} 8^{2} 10^{2}\right]$ cages with each imidazolium ring in adjacent cages and the methylene chain going through the $10 \mathrm{MR}$ that connects them (see below). Because of charge-balance, there must be six fluoride anions per cell, which should occupy the D4R units (see below). In contrast, samples prepared with 5BDMI and 6BDMI, which have been proved to be less effective SDAs compared with 4BDMI, show slightly less than three dications per cell.

To confirm the integrity of the SDAs, ${ }^{13} \mathrm{C}$ MAS NMR was carried out on the as-made samples as shown in Fig. 2. The spectra show resonances close to the expected ranges for each $\mathrm{C}$ atom, suggesting that the organics are essentially intact in all

Table 1 Chemical analysis of as-made zeolites synthesized in this work

\begin{tabular}{|c|c|c|c|c|c|c|c|c|}
\hline SDA & Phase & $\mathrm{C}(\%)$ & $\mathrm{H}(\%)$ & $\mathrm{N}(\%)$ & $\mathrm{C} / \mathrm{N}^{a}$ & $\mathrm{H} / \mathrm{N}^{a}$ & $\mathrm{TG}^{b}$ & Empirical composition $^{c}$ \\
\hline 4BDMI & Si-MTW & 5.71 & 0.93 & 1.57 & $4.24(3.50)$ & $8.23(6.00)$ & $89.09(91.63)$ & $\left|\mathrm{C}_{14} \mathrm{H}_{24} \mathrm{~N}_{4} \mathrm{~F}_{2}\right|_{1.02}\left[\mathrm{SiO}_{2}\right]_{56}$ \\
\hline 4BDMI & Si-STW & 11.46 & 1.24 & 3.78 & $3.54(3.50)$ & $4.56(6.00)$ & $79.08(80.68)$ & $\left|\mathrm{C}_{14} \mathrm{H}_{24} \mathrm{~N}_{4} \mathrm{~F}_{2}\right|_{3.01}\left[\mathrm{SiO}_{2}\right]_{60}$ \\
\hline 4BDMI & Si,Ge-STW & 8.48 & 1.35 & 2.69 & $3.68(3.50)$ & $6.97(6.00)$ & $84.88(86.25)$ & $\left|\mathrm{C}_{14} \mathrm{H}_{24} \mathrm{~N}_{4} \mathrm{~F}_{2}\right|_{2.82}\left[\mathrm{Si}_{0.5} \mathrm{Ge}_{0.5} \mathrm{O}_{2}\right]_{60}$ \\
\hline 5BDMI & Si,Ge-STW & 9.80 & 1.82 & 3.32 & $3.45(3.75)$ & $7.61(6.50)$ & $84.53(82.23)$ & $\left|\mathrm{C}_{15} \mathrm{H}_{26} \mathrm{~N}_{4} \mathrm{~F}_{2}\right|_{2.79}\left[\mathrm{Si}_{0.9} \mathrm{Ge}_{0.1} \mathrm{O}_{2}\right]_{60}$ \\
\hline 6BDMI & Si,Ge-STW & 8.42 & 1.40 & 2.35 & $4.18(4.00)$ & $8.28(7.00)$ & $85.13(86.81)$ & $\left|\mathrm{C}_{16} \mathrm{H}_{28} \mathrm{~N}_{4} \mathrm{~F}_{2}\right|_{2.45}\left[\mathrm{Si}_{0.5} \mathrm{Ge}_{0.5} \mathrm{O}_{2}\right]_{60}$ \\
\hline
\end{tabular}

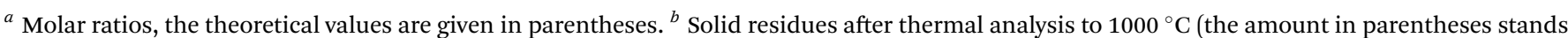

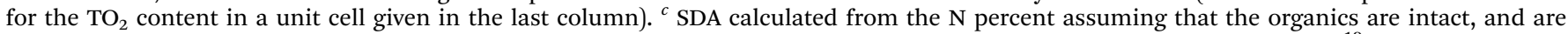

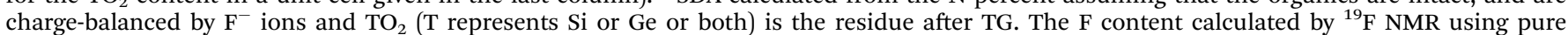

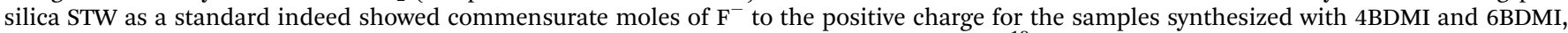
while the 5BDMI sample, however, gave a somewhat higher F content due to impurities (see ${ }^{19} \mathrm{~F}$ NMR in Fig. 3 ). 
the samples. However, there are obvious splittings of several resonances, particularly evident in the case of the pure silica 4BDMI-STW sample, which has the narrowest lines among all the spectra, but are also present in other samples, very clearly in the germanosilicate 5BDMI-STW. Due to the uncertainty about the integrity of the dications that those splitting might induce, we collected ${ }^{1} \mathrm{H}$ and ${ }^{13} \mathrm{C}$ NMR spectra in $\mathrm{D}_{2} \mathrm{O}$ after dissolving the zeolites with the aid of $\mathrm{HF}$ according to our previously reported procedure. ${ }^{5}$ The results (Fig. S5 and $66 \dagger$ ) demonstrate that the dications are intact in the zeolites. Thus, the observed splittings in the solid NMR spectra are attributed to the several possible conformations that are possible in these systems (see computational results below). On the other hand, the broadening of the ${ }^{13} \mathrm{C}$ resonances in the Ge-containing materials may be attributed, at least in part, to a range of different interactions/ conformations depending on the distribution of $\mathrm{Si}$ and $\mathrm{Ge}$ over different crystallographic sites in the germanosilicates.

Thermogravimetric analysis (TGA) combined with derivative thermogravimetry (DTG) shows different patterns for the pure silica and germanosilicate STW zeolites (Fig. S7†). The pure silica STW synthesized with 4BDMI displays a smooth curve having a major weight loss in the range between 400 and $600{ }^{\circ} \mathrm{C}$. The germanosilicate samples, however, exhibit multiple weight loss stages suggesting more complicated chemical changes during the calcination compared to their siliceous counterpart. As recently shown, calcination of organic-containing germanium zeolites can entail complex processes, including the (partial) reduction of the framework to $\mathrm{GeO}$ or metallic $\mathrm{Ge0}$, reoxidation to $\mathrm{GeO}_{2}$ at higher temperature, with the corresponding weight gain, and even $\mathrm{GeO}$ sublimation that would result in weight losses exceeding that of organics and fluoride. ${ }^{20}$ Although this is more likely to happen in small pore germanates, examples were also provided for germanosilicates with larger pores and, in fact, a small weight gaining step in the 800$825{ }^{\circ} \mathrm{C}$ range can be clearly observed in the TGA trace of $4 \mathrm{BDMI}-$ Si,Ge-STW revealing reoxidation of some previously reduced $\mathrm{GeO}_{2}$. From a careful observation of the TG traces, we deem likely that weight gaining processes may occur in similar temperature ranges in all the Si, Ge zeolites shown in Fig. S7, $\uparrow$ but remain unobserved because they overlap with larger weight losing processes. In fact, partial reduction of the framework should be more likely for the bigger dications due to their higher reduction power and the increased difficulty of oxygen diffusion to the zeolite interior. ${ }^{20}$

The ${ }^{29} \mathrm{Si}$ MAS NMR spectra of the silicogermanate zeolites (Fig. S8†) show multiple severely overlapped peaks in the $-90 \mathrm{ppm}$ to $-120 \mathrm{ppm}$ region, which are typical of germanosilicate zeolites. More interesting are the ${ }^{19} \mathrm{~F}$ MAS NMR spectra of the as-made samples shown in Fig. 3. For the as-made germanosilicate zeolites, all exhibit one resonance centered around $-7.2 \mathrm{ppm}$ (4BDMI), -7.4 ppm (5BDMI) and $-7.6 \mathrm{ppm}$ (6BDMI), that we assign to $\mathrm{F}^{-}$located in Ge-containing D4Rs. Similar resonances have been assigned to D4R containing $4 \mathrm{Ge}$ and $4 \mathrm{Si}$ atoms ${ }^{21}$ or both $4 \mathrm{Ge} 4 \mathrm{Si}$ and $5 \mathrm{Si} 3 \mathrm{Ge} .^{22}$ The asymmetrical feature of the spectra may suggest several different environments with similar chemical shifts (possibly corresponding to $\mathrm{F}$ in $\mathrm{Ge}_{x} \mathrm{Si}_{(8-x)}$ with $\left.1<x<8\right)$. Additionally, the spectrum of the

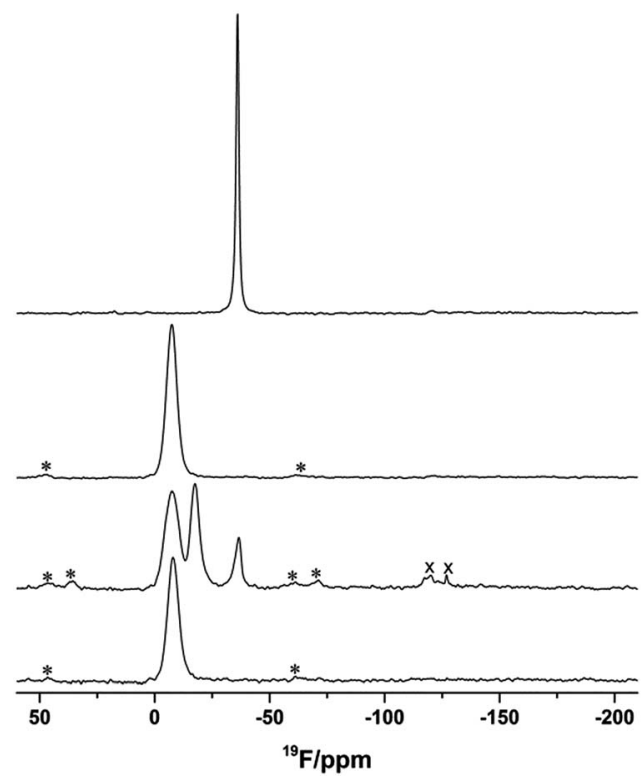

Fig. $3{ }^{19} \mathrm{~F}$ MAS NMR spectra of as-made (germano)silicate STW zeolites (from top to bottom): 4BDMI-Si-STW, 4BDMI-Si,Ge-STW $\left(\mathrm{Ge}_{f}=0.5\right)$, 5BDMI-Si,Ge-STW $\left(\mathrm{Ge}_{f}=0.1\right)$ and 6BDMI-Si,Ge-STW $\left(\mathrm{Ge}_{\mathrm{f}}=0.5\right)$. Spinning side bands and impurities are marked with * and $x$, respectively.

5BDMI-STW (with a $\mathrm{Ge}_{\mathrm{f}}=0.1$ ) contains two additional resonances at -17.3 and -36.5 ppm assigned to $\mathrm{F}^{-}$occluded in $\mathrm{D} 4 \mathrm{R}$ units containing only one Ge or no Ge, respectively, in agreement with the much higher $\mathrm{Si} / \mathrm{Ge}$ ratio of this material. Weak resonances around $-120 \mathrm{ppm}$ in this spectrum are assigned to impurities (possibly $\mathrm{F}$ bonded to penta or hexacoordinated Si in the outer surface of the crystallites). As for the pure silica 4BDMI-STW zeolite, a prominent peak centered around $-36 \mathrm{ppm}$ is close to our previously reported resonance in HPM1 prepared with 2-ethyl-1,3,4-trimethylimidazole $(-35.7 \mathrm{ppm})$, and is consequently assigned to $\mathrm{F}^{-}$in the siliceous D4Rs. ${ }^{5}$

The field emission scanning electron microscope (FE-SEM) images of pure silica and germanosilicate STW samples are shown in Fig. S9. $\dagger$ For 4BDMI-STW zeolites, the morphology varies significantly upon introduction of Ge from aggregated nano-meter oval-shaped particles to micrometer-sized wellfaceted spindle-shaped crystals. A similar morphology was obtained for 6BDMI-STW zeolites with, however, an amorphous phase clearly shown on the surface of the crystals, further confirming the less effective structure-directing ability of this dication. Thus, the introduction of Ge has not only accelerated the crystallization rate but also afforded an interesting influence on the crystal morphologies.

\section{Rietveld refinement of $4 \mathrm{BDMI}-\mathrm{Si}, \mathrm{Ge}-\mathrm{STW}, \mathrm{Ge}_{\mathrm{f}}=\mathbf{0 . 5}$}

Rietveld refinement of the structure of 4BDMI-Si,Ge-STW using synchrotron X-ray powder diffraction data was undertaken using GSAS $^{23}$ under the EXPGUI interface, ${ }^{24}$ and the details are provided as ESI $\dagger$ and the cif file has been deposited with the Cambridge Crystallographic Data Center (CCDC 1578113). Despite the difficulties in dealing with the large and flexible 
dication (see ESI $\dagger$ ), the refinement converged satisfactorily (final $\mathrm{Rp}=3.87 \% \mathrm{Rwp}=5.25 \%$ ) and the refined structure appears reasonable. The average bonds and angles in the framework, provided in Table $\mathbf{S} 3, \dagger$ are close to the expected values for this class of materials, despite the lack of distance restrains in the final refinement stage. Very interestingly, the refined occupancies of Ge and Si in each site (Table 2) indicate a rather large preference for Ge to occupy positions in the vertices of the D4R (T1 to T4), while site T5 is occupied significantly more by $\mathrm{Si}$. The refined overall Ge molar fraction $\left(\mathrm{Ge}_{\mathrm{f}}=\right.$ 0.536), which was not restrained, was close but a bit larger than the nominal value in the synthesis gel $\left(\mathrm{Ge}_{\mathrm{f}}=0.5\right)$. The refined position of the fluoride anion was very close to the center of the D4R (only $0.004 \AA$ apart from the geometrical center of the $8 \mathrm{~T}$ sites at the vertices of the cage) with distances to the vertices in the 2.69-2.77 ̊ range (average $2.72 \AA$ ).

\section{Computational results}

(A) Structure-directing effect of the $\boldsymbol{n B D M I}$ dications. Once we know the structure-directing behavior of the three $n$ BDMI cations from our synthetic study, we performed a computational study in order to understand the observed differences. $4 \mathrm{BDMI}$ dications were the first studied. They were initially loaded in the STW framework through Monte-Carlo simulations: results showed that $4 \mathrm{BDMI}$ is located siting each imidazolium ring in adjacent STW cavities with the C4 spacer running through the 10-rings connecting the cavities, giving a maximum loading of 3 SDAs per STW unit cell (in perfect agreement with the experimental observations, see Table 1). Due to the asymmetry of the imidazolium rings, which have both methyl substituents at one side, and the asymmetry of the STW cavities (see Fig. S11† for a detailed explanation of the STW framework and cavities), two different orientations of these rings confined within the STW cavities are possible: in one of them, the central methyl group attached to $\mathrm{C}(\mathrm{C}(\mathrm{e})$ in Fig. 2) points to an 8-ring of the cavity ( $8 R$ configuration), while in the other, which is related by $180^{\circ}$ rotation of the ring plane, the methyl group attached to $\mathrm{C}(\mathrm{e})$ points to the 10-ring ( $10 R$ configuration). As a result, three different orientations for 4BDMI were manually built ( $10 R 10 R$, $8 R 8 R$ and $8 R 10 R$, see Fig. 4), and the most stable locations for each were determined by the simulated annealing-DFT protocol previously explained (Table 3).The most stable configuration of 4BDMI dications hosted in the STW framework involves the two imidazolium rings in the $8 R$ configuration $(8 R 8 R$, with an interaction energy of $1170.7 \mathrm{kcal} \mathrm{mol}^{-1}$ per STW u.c., as

Table 2 . Rietveld refined Ge molar fractions per crystallographic site in 4BDMI-Ge,Si-STW (overall $\mathrm{Ge}_{f}=0.536$ )

\section{T site}

$\mathrm{Ge}_{\mathrm{f}}$
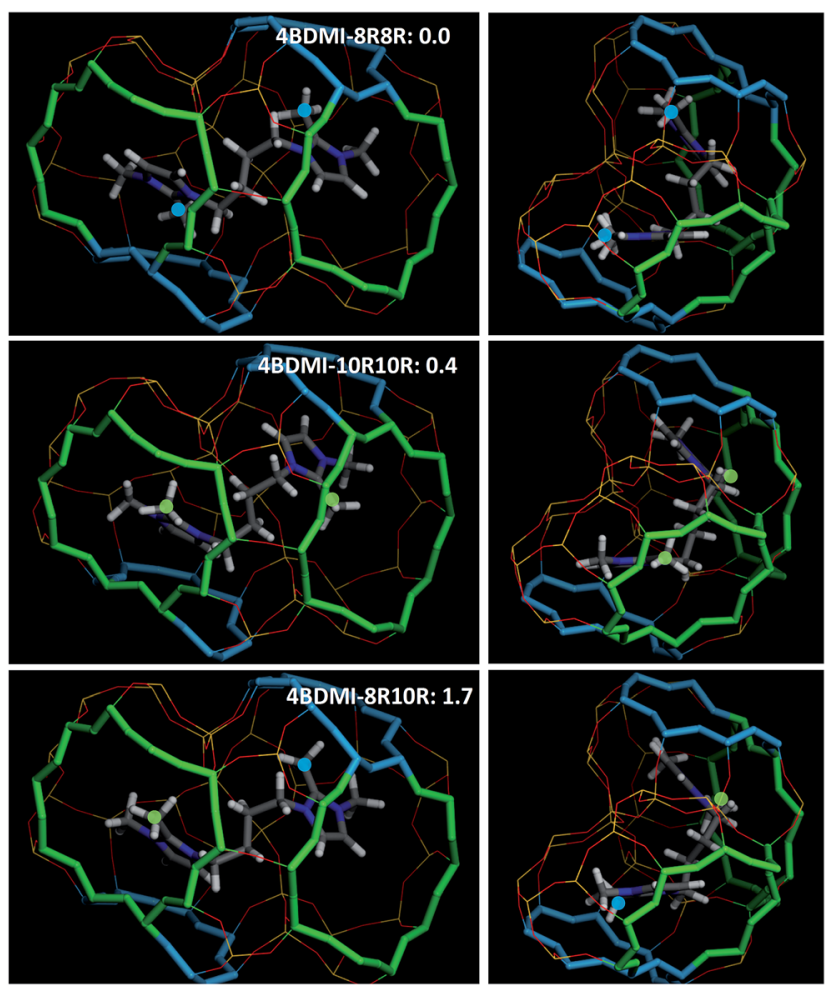

Fig. 4 Two views (right and left) of the location of 4BDMl dications in the different configurations, showing also the relative energies with respect to the most stable case; $10 R$ and $8 R$-windows are highlighted as green and blue sticks, respectively. Methyl $C(e)$ atoms pointing to $10 R$ or $8 R$-windows are highlighted with green or blue balls, respectively.

Table 3 Relative Energies (Rel. E.) (with respect to the most stable case, highlighted in bold) and Interaction Energies (Int. E., between brackets) for the three dications used experimentally in this work and in the different configurations, as calculated by DFT+D (in kcal mol $^{-1}$ per STW unit cell)

\begin{tabular}{lll}
\hline SDA & Orientation & Rel. E. (Int. E.) \\
\hline 4BDMI & $8 R 8 R$ & $\mathbf{0 . 0}(-\mathbf{1 1 7 0 . 7})$ \\
& $10 R 10 R$ & $0.43(-1170.3)$ \\
5 BDMI & $8 R 10 R$ & $1.70(-1169.0)$ \\
& $8 R 8 R$ & $\mathbf{0 . 0}(-\mathbf{1 1 6 4 . 2})$ \\
6BDMI & $10 R 10 R$ & $5.62(-1158.6)$ \\
& $8 R 10 R$ & $2.48(-1161.7)$ \\
& $8 R 8 R$ & $16.7(-1132.9)$ \\
& $10 R 10 R$ & $\mathbf{0 . 0}(-\mathbf{1 1 4 9 . 6})$ \\
& $8 R 10 R$ & $1.45(-1148.2)$
\end{tabular}

determined by DFT+D). Nevertheless, the energy difference with the other two configurations is very low $(0.40$ and $1.70 \mathrm{kcal} \mathrm{mol}^{-1}$ for $10 R 10 R$ and $8 R 10 R$ configurations, respectively, see Table 3), and hence all of them should be feasible. These small energy differences suggest that the STW cavities are large enough to host the imidazolium rings in the two possible orientations, and the stability of each configuration will depend on the fitting of the spacer as a function of its length. Fig. 4 shows the locations of the 4BDMI dications (in the three stable 
configurations), where we observe that the 4BDMI dications span two consecutive STW cavities, with the spacer running through the 10-ring connecting the two cavities, and folding in such a way as to follow the helicoidal pattern of the 10-ring channel (see Fig. 4-right); indeed, the angles between the planes of the two imidazolium rings are close to $60^{\circ}$ (the same as the angle between cavities). As expected due to the non-chiral nature of $4 \mathrm{BDMI}$, the host-guest interaction between the 4BDMI dication and the other STW enantiomorph (in the $P 6_{5} 22$ space group) was exactly the same, but the SDAs folded in the opposite handedness to follow the corresponding helicoidal STW channel.

We next studied the 5BDMI cation through the same methodology, and in the same three orientations. Again the most stable configuration involves having both imidazolium rings in the $8 R$ configuration ( $8 R 8 R$, see Table 3$)$ (Fig. 5), giving an interaction energy of $-1164.2 \mathrm{kcal} \mathrm{mol}^{-1}$ per STW u.c., which is lower than that of 4BDMI. In this case, the longer length of the spacer involves a reduced space to adjust the particular conformations, and as a consequence, the differences in the stability between the different configurations are higher (with a relative energy of $5.62 \mathrm{kcal} \mathrm{mol}^{-1}$ for the $10 R 10 R$ configuration, see Fig. S12†).

Finally the longest 6BDMI dication was studied. In contrast to the previous cases, the most stable configuration involves now the $10 R 10 R$ configuration (Table 3 ), rendering the lowest interaction energy of $1149.6 \mathrm{kcal} \mathrm{mol}^{-1}$ per STW u.c., which is rather lower than that of 4BDMI (despite the larger number of interacting atoms in 6BDMI). The even longer alkyl chain length involves a larger difference in the stability between the different configurations to accommodate the large spacer, with energy differences up to $16.7 \mathrm{kcal} \mathrm{mol}^{-1}$ for the $8 R 8 R$ configuration which was the most stable one for the shorter dications. Hence, because of its longer length, the spacer hosted between the two STW cavities has to fold strongly to fit the orientation of the imidazolium rings with the $\mathrm{C}(\mathrm{e})$ methyl groups pointing to the $8 R$ side of the cavity, resulting in strong destabilization through a conformational energy penalty (see Fig. S12†).

In sum, our computational results suggest that, while the 1,2-dimethyl-imidazolium rings are suitable templates for the cavities of the STW framework, the spacers with alkyl chains of 4, 5 or 6 methylenes linking the rings have the appropriate size to span two consecutive STW cavities. Interestingly, the high
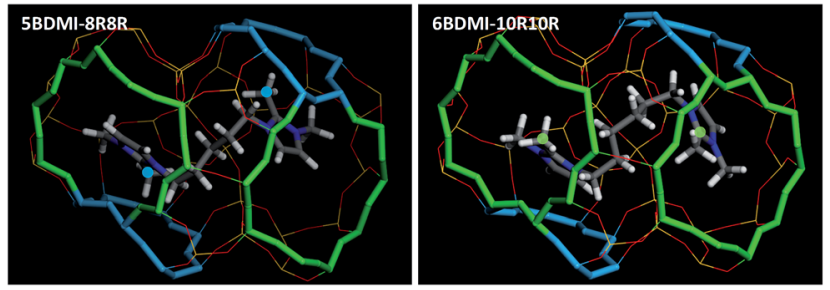

Fig. 5 Location of 5BDMI (left) and 6BDMI (right) dications in the most stable configuration ( $8 R 8 R$ for $5 \mathrm{BDMI}$ and $10 R 10 R$ for $6 \mathrm{BDMI}$ ); $10 R$ and $8 R$-windows are highlighted as green and blue sticks, respectively. Methyl $C(e)$ atoms pointing to $10 R$ or $8 R$-windows are highlighted with green or blue balls, respectively. ratio of freely-rotating single bonds in the alkyl chain allows this type of SDA to fold in a particular conformation which follows the helicoidal pattern of the STW channels, thus explaining the ability of these SDAs to template the formation of this framework. The interaction energies of the host-guest systems suggest that the structure-directing efficiency of the three dications should follow the decreasing order 4BDMI > 5BDMI > 6BDMI, which is in perfect agreement with the experimental observations, and shows that the best fit is achieved for the $\mathrm{C} 4$ spacer. Indeed, repetition of the calculations by molecular mechanics methodologies (both using cvff or Dreiding forcefields) gives the same energy trends (see Table $\mathrm{S} 4 \dagger$ in the ESI $\dagger$ ); cvff calculations show a similar contribution from van der Waals and electrostatic terms to the overall interaction energy. On the other hand, the better fit of the $\mathrm{C} 4$ spacer implies that, from a thermodynamic point of view, the three possible configurations of $4 \mathrm{BDMI}$ could occur during crystallization (being the $8 R 8 R$ and $10 R 10 R$ configurations slightly more likely), whilst the $8 R 8 R$ configuration is particularly unstable for $6 \mathrm{BDMI}$ due to the longer length of the spacer.

(B) Calculation of the ${ }^{13} \mathrm{C}$ NMR spectra. We then calculated theoretically the ${ }^{13} \mathrm{C}$ NMR spectra of the three $n \mathrm{BDMI}$ dications in the different configurations (Table 4). First we analyzed the results for the $4 \mathrm{BDMI}$ dications, and compared them with the experimental ${ }^{13} \mathrm{C}$ NMR spectrum of 4BDMI-Si-STW, which shows well-resolved resonances (Fig. 2-bottom). The theoretical ${ }^{13} \mathrm{C}$ chemical shifts of $4 \mathrm{BDMI}$ in the different configurations show several differences brought about by the particular orientation of the imidazolium rings (in $10 R$ or $8 R$ configurations). Of particular relevance is the case of the $\mathrm{C}(\mathrm{e})$ shift, which is around $9.0 \mathrm{ppm}$ when the methyl group points to the $8 R$, and around 6.3 when it points to the $10 R$; similarly, the shift of $\mathrm{C}(\mathrm{f})$ is also particularly affected by the SDA configuration, being around $50 \mathrm{ppm}$ in the $8 R 8 R$ configuration and around $53 \mathrm{ppm}$ in the $10 R 10 R$ configuration. Interestingly, such splittings of the $\mathrm{C}(\mathrm{e})$ and $\mathrm{C}(\mathrm{f})$ resonances are nicely comparable with the two signals at 9.0 and $7.6 \mathrm{ppm}(\mathrm{C}(\mathrm{e}))$ and several other centered at 50 and $48 \mathrm{ppm}(\mathrm{C}(\mathrm{f}))$ of the 4BDMI-Si-STW experimental spectrum. $\mathrm{C}(\mathrm{g})$ gives a group of several resonances between 23.3 and 30.2 ppm, while C(d) gives signals between 35 and 38 ppm; in this case, it is difficult to unambiguously assign these signals to a particular orientation of the imidazolium rings. Finally, $\mathrm{C}(\mathrm{b}, \mathrm{c})$ gives signals at 122 and 127 ppm, and C(a) gives signals between 143 and 145 ppm, which again cannot be clearly used to identify the distinct configurations. Hence, we can use the splitting of the two ${ }^{13} \mathrm{C}$ NMR signals of $\mathrm{C}(\mathrm{e})$ and $\mathrm{C}(\mathrm{f})$ to identify the presence of the 4BDMI dications in the different configurations; we note that the theoretical chemical shifts are not exactly the same as the experimental ones, but the overall trends are in good agreement. By comparing with the experimental spectrum of 4BDMI-Si-STW, we can clearly observe that both configurations $(10 R 10 R$ and $8 R 8 R)$ should be present in the material, and even the $10 R 8 R$ configuration (this mixed configuration is indistinguishable from a mixture of the pure $10 R 10 R$ and $8 R 8 R$ ones); this is in good agreement with the very similar stabilities observed for the three configurations (see Table 3). Therefore, our computational DFT study indicates that ${ }^{13} \mathrm{C}$ NMR could be 
Table $4{ }^{13} \mathrm{C}$ NMR chemical shifts for the three SDA dications occluded within the STW framework in the different configurations. Labels of the C atoms are those in Fig. 2

\begin{tabular}{|c|c|c|c|c|c|c|c|c|c|c|}
\hline SDA & Config. & Rel. E. & $\mathrm{a}$ & $\mathrm{b}$ & $\mathrm{c}$ & $\mathrm{f}$ & d & $\mathrm{g}$ & $\mathrm{h}$ & e \\
\hline \multirow[t]{6}{*}{ 4BDMI } & \multirow[t]{2}{*}{$8 R 8 R$} & \multirow[t]{2}{*}{0.0} & 143.6 & 122.7 & 124.8 & 50.3 & 37.0 & 26.2 & - & 8.5 \\
\hline & & & 145.0 & 123.5 & 122.3 & 49.6 & 36.7 & 23.3 & - & 9.4 \\
\hline & \multirow[t]{2}{*}{$10 R 10 R$} & \multirow[t]{2}{*}{0.4} & 143.2 & 127.2 & 123.2 & 52.9 & 35.0 & 30.2 & - & 6.3 \\
\hline & & & 143.6 & 127.2 & 123.3 & 52.8 & 37.6 & 30.1 & - & 6.2 \\
\hline & \multirow{2}{*}{$8 R 10 R$} & \multirow[t]{2}{*}{1.7} & 143.6 & 127.5 & 122.4 & 52.7 & 38.3 & 29.3 & - & 6.4 \\
\hline & & & 143.4 & 121.9 & 125.4 & 52.9 & 37.9 & 27.9 & - & 7.9 \\
\hline \multirow[t]{6}{*}{ 5BDMI } & \multirow[t]{2}{*}{$8 R 8 R$} & \multirow[t]{2}{*}{0.0} & 144.6 & 122.9 & 120.4 & 51.0 & 38.7 & 26.9 & 23.5 & 11.3 \\
\hline & & & 144.6 & 122.8 & 120.4 & 51.0 & 38.7 & 26.9 & - & 11.4 \\
\hline & \multirow[t]{2}{*}{$10 R 10 R$} & \multirow[t]{2}{*}{5.6} & 142.9 & 127.8 & 124.1 & 55.6 & 35.9 & 27.4 & 22.2 & 7.9 \\
\hline & & & 143.0 & 128.2 & 123.6 & 55.6 & 36.0 & 27.5 & - & 7.9 \\
\hline & \multirow[t]{2}{*}{$8 R 10 R$} & \multirow[t]{2}{*}{2.5} & 143.1 & 128.2 & 124.0 & 55.8 & 36.3 & 27.4 & 22.6 & 7.9 \\
\hline & & & 144.6 & 122.6 & 120.6 & 51.2 & 38.4 & 27.1 & - & 11.1 \\
\hline \multirow[t]{6}{*}{ 6BDMI } & \multirow[t]{2}{*}{$8 R 8 R$} & \multirow[t]{2}{*}{16.7} & 143.2 & 122.7 & 125.8 & 55.8 & 37.9 & 30.3 & 27.5 & 10.4 \\
\hline & & & 143.3 & 122.9 & 125.5 & 55.8 & 37.7 & 30.1 & 27.8 & 10.7 \\
\hline & \multirow[t]{2}{*}{$10 R 10 R$} & \multirow[t]{2}{*}{0.0} & 142.2 & 125.2 & 126.6 & 51.8 & 38.4 & 28.7 & 29.8 & 9.5 \\
\hline & & & 143.3 & 127.0 & 124.3 & 52.7 & 36.0 & 31.0 & 26.9 & 7.9 \\
\hline & \multirow[t]{2}{*}{$8 R 10 R$} & \multirow[t]{2}{*}{1.5} & 141.6 & 126.2 & 127.0 & 52.8 & 38.7 & 28.2 & 30.2 & 10.5 \\
\hline & & & 143.5 & 121.5 & 125.7 & 52.6 & 37.9 & 29.1 & 27.3 & 8.1 \\
\hline
\end{tabular}

used to identify different configurations of the guest species occluded in these zeolite materials. The reason for the splitting of the $\mathrm{C}(\mathrm{e})$ might be associated with the different confinement provided by the STW cavities when the $\mathrm{C}(\mathrm{e})$ methyl group is in one or the other side of the asymmetric cavity (see Fig. 4). Indeed, calculation of the Radial Distribution Function between $\mathrm{C}(\mathrm{e})$ atoms and $\mathrm{O}$ zeolite frameworks (during MD simulations) (see Fig. S13 $\dagger$ ) indicates a much stronger confinement when $\mathrm{C}(\mathrm{e})$ points to the $8 R$ (indicated by the high intensity of the peak at $3.8 \AA$ in the $8 R 8 R$ configuration), due to the smaller cross section in this side of the STW cavity. Interestingly, the presence of Ge in the material causes a change of the relative intensities of the resonances corresponding to the distinct configurations; keeping in mind that these ${ }^{13} \mathrm{C}$ NMR spectra are not quantitative, it seems that the configuration $10 R 10 R$ prevails in the case of the germanosilicate material, which must be associated with the larger size of Ge.

We next studied the system with 5BDMI. After careful inspection of the NMR theoretical chemical shifts and comparison with the experimental ones, we again realize that the same two $\mathrm{C}(\mathrm{e})$ and $\mathrm{C}(\mathrm{f})$ resonances can be used to identify the $10 R$ and $8 R$ orientations of the imidazolium rings in the STW cavities, with $\mathrm{C}(\mathrm{e})$ giving signals at 11 and $8 \mathrm{ppm}$ in $8 R$ and $10 R$ orientations, respectively, and $\mathrm{C}(\mathrm{f})$ giving signals at 51 and $55 \mathrm{ppm}$ in $8 R$ and $10 R$ orientations, respectively. Comparison with the experimental ${ }^{13} \mathrm{C}$ NMR spectrum suggests a more abundant presence of the $8 R$ orientation for the imidazolium rings (because of the higher intensity of the resonances at 10.5 and $48.0 \mathrm{ppm}$ assigned to the $8 R$ orientation compared with that of the resonances at 8.9 and $50.9 \mathrm{ppm}$ assigned to the $10 R$ orientation). We note again that the intensities are not an exact indication of the concentration of species, but nevertheless the trends are clear. This is again in very good agreement with the higher stability of the $8 R 8 R$ configuration (Table 3 ).
Finally, in the case of the 6BDMI-STW system, the trends are not as clear as in the previous cases; indeed, the resolution of the ${ }^{13} \mathrm{C}$ NMR bands is much lower in this case (see Fig. 2-top). $\mathrm{C}(\mathrm{e})$ in the $8 R 8 R$ configuration gives a theoretical signal at higher shifts (10.5 ppm) (Table 3), although in this case the difference is not as clear as for 5BDMI and 6BDMI. The experimental resonance corresponding to this $\mathrm{C}$ atom centered at $9.1 \mathrm{ppm}$ seems to be asymmetric, with a slightly higher intensity at lower chemical shift; this could be an indication of a stronger presence of the $10 R 10 R$ configuration, in good agreement with the energetic results (Table 3). However, such assignment should give a higher intensity of the $\mathrm{C}(\mathrm{d})$ resonance (two bands at around $50 \mathrm{ppm}$ ) at lower shift, while the opposite is found in the experimental spectrum. Nonetheless, after inspection of a large number of calculations that we have carried out, we have observed that such splitting of the $\mathrm{C}(\mathrm{d})$ resonance is due to the particular folding of the long C6 spacer which has a very high degree of motion, and hence is not directly related to the orientation of the imidazolium ring. Therefore, although here is not as clear as in the previous cases, by combining with the energy results we tentatively propose a more abundant presence of the $10 R 10 R$ configuration for $6 \mathrm{BDMI}$ in the STW framework, which is directly caused by the length of the C6 spacer.

(C) Exploration of asymmetric dications. Of course the symmetric nature of the $n$ BDMI dications used experimentally in this work implies that conformations with the alkyl chain folded helicoidally in one or the opposite handedness (following the two STW enantiomorphic space groups) are energetically equivalent, and therefore a mixture of the two enantiomorphic crystals will be obtained. Introducing some asymmetry in the alkyl chain might break the energy degeneracy when folding the SDAs in the two directions, resulting in the crystallization of a particular enantiomorphic STW crystal, as very recently shown by Brand et al. ${ }^{2}$ We thought our 4BDMI dication could be a good starting point for the generation of enantiospecific SDAs since, 
on the one hand, 4BDMI, because of the appropriate length of the linker, appears to be a notable SDA for STW and, on the other hand, the not fully substituted imidazolium rings could still afford some room favoring accommodation in adjacent cages even after being modified to become asymmetric. Thus, we have designed SDAs based on 4BDMI, by introducing 1 or 2 methyl atoms in different positions and configurations, giving different enantiomers and diastereoisomers, and we determined (by molecular mechanics calculations using both cvff and Dreiding forcefields) the interaction energy (studying just one SDA spanning two STW cavities) by simulated annealing calculations, starting from the different possible orientations of the imidazolium rings. For the sake of completeness, we have also performed the same study for the tetramethylimidazolium derivatives used by Brand et al., and also for other tetramethylimidazolium derivatives related to our SDAs. We calculated the interaction energies of the different enantiomers and the STW framework (in the $P 6_{1} 22$ space group), as well as the energy difference between the two molecular enantiomers $(\Delta E$ in Table 5), using the two different force-fields. Discussion of the results will be based on the energy results obtained with cvff, but similar trends are observed for results obtained with Dreiding (except for a specific case, 4BDMI-C2).

As expected, 4BDMI displays the same interaction energy in $P 6_{1} 22$ and $P 6_{5} 22$ enantiomorphic STW crystals (entry 1 in Table 5 ). The asymmetry induced by additional methyl groups in the alkyl chain does indeed lead to differences in the interaction energies between the two enantiomers and the $P 6_{1} 22$ STW framework. We first focus on the SDAs derived from those used experimentally in this work, with two methyl groups in the imidazolium rings, and with 1 or 2 methyl groups attached to the C4 spacer (in the two possible positions). Chiral SDAs with a methyl group on $\mathrm{C}(\mathrm{f})$ (see Fig. 2 for labels, positions 1 or 4 of the spacer, 4BDMI-C1 with one methyl group, entry 2, and 4BDMI-C1,4 with two, entry 4) fit better within the STW framework (in terms of a higher interaction energy) than those where the methyl group is attached on positions 2 (or 3) (compare energies of entries 2 and 3 and energies of entries 4 and 5) (Table 5). This is so because the methyl groups in position $2 / 3$ develop steric repulsions with the 10-rings. As a consequence, the energy difference between the enantiomers is higher when the methyl groups are in these central (2 and 3) positions (2.2 vs. 1.9 for SDAs with one methyl group in position 2 or 1 , entries 3 and 2, respectively, or 8.9 vs. 5.2 for SDAs with two methyl groups in positions 2,3 or 1,4, entries 5 and 4, respectively). As expected, an increase of the number of methyl groups in the spacer enhances the enantiomeric energy differences. The highest energy difference is observed for 4BDMI-C2,3 (entry 5), but at the same time this cation also displays the lowest interaction energy (119.2 for $R R$ ), and hence one would expect a decreased ability to direct the crystallization of the STW framework. In certain configurations (4BDMI-C1,4-RS and 4BDMI-C2,3-RS), meso compounds are obtained (which are achiral), and the energy results indeed confirm no enantioselection (enantiomeric energy differences of 0 were found, as expected).

We have also studied similar SDA cations with methyl groups in the C4 spacer, but with imidazolium rings with four methyl groups, since these rings were predicted to fit best within the STW cavities. These SDAs (4BPerMI-C1, 4BPerMI-C2, 4BPerMI$\mathrm{C} 1,4$ and 4BPerMI-C2,3, entries 6 to 9 in Table 5) are abbreviated using a code that includes " $4 \mathrm{~B}$ " (for the tetramethylene linker), "PerMI" (for the permethylated imidazolium moieties) and " $\mathrm{C} n$ " to indicate the position $n$ of the methyl substituent along the linker. Thus, (1-methylbutane-1,4-diyl)bis(2,3,4,5tetramethylimidazolium) is abbreviated as 4BPerMI-C1, since it contains a methyl group at the first carbon of the tetramethylene linker. ${ }^{2,25}$ We note that the derivative with two methyl groups in positions 1 and 4 (entry 8, 4BPerMI-C1,4) displays the highest interaction energy with the framework (139.2) while yielding a large enantiomeric energy difference $\left(6.1 \mathrm{kcal} \mathrm{mol}^{-1}\right.$ in favour of the $S S$ enantiomer). These results are confirmed when using the Dreiding forcefield for the calculation of the interaction energies, which again predicts this as the best SDA for the STW framework (in terms of the highest interaction energy, 117.1) while displaying a very large enantiomeric energy difference (12.3 in favor of the $S S$ enantiomer). In this case, both enantiomers of the 2,3-isomer show a very bad adjustment with the framework, resulting in a very low interaction energy and negligible enantiomeric energy differences since none of the enantiomers fits within the STW framework (entry 9).

We then studied a different spacer based on a trans-cyclopropane unit, the same as Davis and co-workers have recently used successfully for the enantio-enrichment of the STW germanosilicate. ${ }^{2}$ In this case, the cvff force-field was not appropriate to model this structural unit (the cyclopropane ring broke upon geometry optimization), and hence we have used only the Dreiding forcefield. When four-methyl-imidazolium rings were used, energy results showed an enantiomeric energy difference of $7.1 \mathrm{kcal} \mathrm{mol}^{-1}$ per SDA (in favor of the $R R$ enantiomer, the same as reported by Davis). This energy difference is in the range of those found previously with our dimethyl-derivatives (entry 4, 4BDMI-C1,4 entry 5, 4BDMI-C2,3, and entry 8, 4BPerMI-C1,4, with energy differences of 7.3, 12.7 and $12.3 \mathrm{kcal} \mathrm{mol}^{-1}$, respectively, as determined using Dreiding), suggesting that these would also enable an enantio-enrichment of the STW zeolite. Surprisingly, using the same spacer with the chiral cyclopropane unit but the imidazolium rings with just two (rather than four) methyl groups notably reduced the enantiomeric energy difference (from 7.1 to $0.8 \mathrm{kcal} \mathrm{mol}^{-1}$ per SDA), which might be caused by the smaller size of the imidazolium rings siting in the cavities enabling a better adjustment to the particular handedness of the STW framework $\left(P 6_{1} 22\right)$ depending on the orientation of the imidazolium rings.

All these results suggest that 4BDMI-C1,4 and 4BPerMI-C1,4 would represent the best candidates to achieve enantioselection during crystallization of the STW framework, even in pure silica form, since these combine a high enantiomeric energy difference and the highest interaction energy (137.3 and $139.2 \mathrm{kcal} \mathrm{mol}{ }^{-1}$, as determined by cvff), which are indeed slightly higher than that of the 4BDMI cation (135.5 kcal mol${ }^{-1}$ ) that experimentally worked for the synthesis of pure silica STW (Fig. S14 $\uparrow$ shows images of the most stable configurations of $S S$ 4BDMI-C1,4 and $R R$-4BDMI-C1,4 in STW). Unfortunately, we have not been able to synthesize these dications. 
Table 5 Interaction Energies (I.E.) and energy differences $(\Delta E)$ (in $\mathrm{kcal} \mathrm{mol}^{-1}$ per SDA) between the two enantiomers using different force-fields. For 4BDMI-C1,4, 4BDMI-C2,3, 4BPerMI-C1,4 and 4BPerMI-C2,3 cases, only the RR/SS diastereoisomers are considered (RS/SR are achiral meso forms). Unless specified (entry 1 ), the $P 6_{1} 22$ STW framework was used

\begin{tabular}{|c|c|c|c|c|c|c|c|}
\hline \multirow[b]{2}{*}{ Entry } & \multirow[b]{2}{*}{ SDA } & \multirow{2}{*}{$\begin{array}{l}\text { Structural } \\
\text { formula }\end{array}$} & \multirow[b]{2}{*}{ Enant } & \multicolumn{2}{|l|}{ cvff } & \multicolumn{2}{|c|}{ Dreiding } \\
\hline & & & & I.E. & $\Delta E$ & I.E. & $\Delta E$ \\
\hline 1 & 4BDMI & & $\begin{array}{l}P 6_{1} 22 \\
P 6_{5} 22\end{array}$ & $\begin{array}{l}-135.5 \\
-135.5\end{array}$ & 0.0 & $\begin{array}{l}-16.1 \\
-16.1\end{array}$ & 0.0 \\
\hline 2 & 4BDMI-C1 & & $\begin{array}{l}R \\
S\end{array}$ & $\begin{array}{l}-137.7 \\
-135.8\end{array}$ & $1.9(R)$ & $\begin{array}{l}-48.9 \\
-46.4\end{array}$ & $2.5(R)$ \\
\hline 3 & 4BDMI-C2 & & $\begin{array}{l}R \\
S\end{array}$ & $\begin{array}{l}-132.6 \\
-130.4\end{array}$ & $2.2(R)$ & $\begin{array}{l}-43.7 \\
-47.1\end{array}$ & $3.4(S)$ \\
\hline 4 & 4BDMI-C1,4 & & $\begin{array}{l}R R \\
S S\end{array}$ & $\begin{array}{l}-132.1 \\
-137.3\end{array}$ & $5.2(S S)$ & $\begin{array}{l}-33.8 \\
-41.1\end{array}$ & $7.3(S S)$ \\
\hline 5 & 4BDMI-C2,3 & & $\begin{array}{l}R \\
S\end{array}$ & $\begin{array}{l}-119.2 \\
-110.3\end{array}$ & $8.9(R R)$ & $\begin{array}{l}-24.4 \\
-11.7\end{array}$ & $12.7(R R)$ \\
\hline 6 & 4BPerMI-C1 & & $\begin{array}{l}R \\
S\end{array}$ & $\begin{array}{l}-137.8 \\
-137.9\end{array}$ & $0.1(S)$ & $\begin{array}{l}-94.7 \\
-99.0\end{array}$ & $4.3(S)$ \\
\hline 7 & 4BPerMI-C2 & & $\begin{array}{l}R \\
S\end{array}$ & $\begin{array}{l}-119.4 \\
-123.3\end{array}$ & $4.0(S)$ & $\begin{array}{l}-56.3 \\
-58.3\end{array}$ & $2.0(S)$ \\
\hline 8 & 4BPerMI-C1,4 & & $\begin{array}{l}R R \\
S S\end{array}$ & $\begin{array}{l}-133.1 \\
-139.2\end{array}$ & $6.1(S S)$ & $\begin{array}{l}-104.8 \\
-117.1\end{array}$ & $12.3(S S)$ \\
\hline 9 & 4BPerMI-C2,3 & & $\begin{array}{l}R R \\
\mathrm{SS}\end{array}$ & $\begin{array}{l}-97.0 \\
-96.2\end{array}$ & $0.8(R R)$ & $\begin{array}{l}+9.2 \\
+9.1\end{array}$ & $0.1(S S)$ \\
\hline 10 & 4BDMI-cycpr & & $\begin{array}{l}\mathrm{RR} \\
S S\end{array}$ & - & - & $\begin{array}{l}-38.5 \\
-37.7\end{array}$ & $0.8(R R)$ \\
\hline 11 & Davis & & $\begin{array}{l}R R \\
S S\end{array}$ & - & - & $\begin{array}{l}-49.3 \\
-42.2\end{array}$ & $7.1(R R)$ \\
\hline
\end{tabular}

\section{Conclusions}

Organic dications based on 1,2-dimethylimidazolium moieties separated by linear linkers of 4, 5 or 6 methylene groups show structure-direction towards the chiral STW zeolite as racemic conglomerates with a strength that depends on the length of the linker and the chemical composition of the synthesis gel. The strongest specificity is displayed by $4 \mathrm{BDMI}$, i.e., the dication with a tetramethylene chain as a linker. This dication works even for pure silica compositions. When the length of the linker 
increases, the specificity towards STW decreases and for 6BDMI (with the hexamethylene linker) only in the presence of Ge could this zeolite be obtained (MTW appearing otherwise).

For 4BDMI and pure silica compositions, when the synthesis gel is diluted MTW appears first but transforms in situ into STW. This is the third example of a zeolite transformation from a denser and less strained zeolite (MTW) to a less dense and more strained one (STW) and reveals significant stabilization of the STW silica framework by fluoride and the dication. This transformation occurs also with 5BDMI (although more slowly). The systems obey the Villaescusa rule, with STW being favored over MTW at higher concentrations of the synthesis gels.

Rietveld refinement of a STW zeolite prepared with 4BDMI and $\mathrm{Ge}_{\mathrm{f}}=0.5$ reveals a preferential sitting of Ge in the D4R, especially on sites T1 and T2. A single but asymmetric ${ }^{19} \mathrm{~F}$ NMR resonance around $-7 \mathrm{ppm}$ is assigned to fluoride occluded in D4R units containing between 2 and 7 Ge atoms.

Molecular simulations of the different dications in STW agree with the experimental findings, as the stabilization of the framework decreases when the length of the linker increases. In addition, comparison of theoretical and experimental ${ }^{13} \mathrm{C}$ NMR results allowed us to identify different configurations of the dications occluded within the STW framework, with different relative populations as a function of the linker length and the cell composition; in fact, the reasonable explanation provided by our computational study of the ${ }^{13} \mathrm{C}$ NMR splittings observed experimentally validates our theoretical approach. A virtual exploration of different derivatives of 4BDMI with methyl groups added in the linker reveals that the dimethylated derivatives in positions 1 and 4 should afford the enantioselective crystallization of STW, even as a pure silica material. By contrast, a cyclopropane moiety similar to that recently reported by Brand et al. but with dimethylated rather than tetramethylated imidazolium units would not be able to impose its chirality to the crystallizing STW.

\section{Conflicts of interest}

There are no conflicts to declare.

\section{Acknowledgements}

The authors acknowledge financial support by the Spanish Ministry of Economy and Competitiveness (projects MAT-201565767-P and MAT2015-71117-R). P. L. is grateful to the China Scholarship Council (CSC) for a fellowship and to the Dalian Institute of Chemical Physics for permission to leave. We are also indebted to the ERSF (Grenoble) and the BM25 Spline staff, particularly to G. Castro and A. Serrano and to L. A. Villaescusa (Valencia) for help in collecting the synchrotron XRD data and for helpful comments and suggestions. Centro Técnico Informático-CSIC is acknowledged for running the calculations, and Accelrys for providing the computational software.

\section{Notes and references}

1 J. M. Newsam, M. M. J. Treacy, W. T. Koetsier and C. B. D. Gruyter, Proc. R. Soc. London, Ser. A, 1988, 420, 375-405.

2 S. K. Brand, J. E. Schmidt, M. W. Deem, F. Daeyaert, Y. Ma, O. Terasaki, M. Orazov and M. E. Davis, Proc. Natl. Acad. Sci. U. S. A., 2017, 114, 5101-5106.

3 A. Rojas, E. Martinez-Morales, C. M. Zicovich-Wilson and M. A. Camblor, J. Am. Chem. Soc., 2012, 134, 2255-2263.

4 Forcite and CASTEP modules, BIOVIA Materials Studio R2, 2017.

5 A. Rojas, O. Arteaga, B. Kahr and M. A. Camblor, J. Am. Chem. Soc., 2013, 135, 11975-11984.

6 Sorption Module, BIOVIA Materials Studio R2, 2017.

7 J. P. Perdew, K. Burke and M. Ernzerhof, Phys. Rev. Lett., 1996, 77, 3865-3868.

8 S. J. Clark, M. D. Segall, C. J. Pickard, P. J. Hasnip, M. J. Probert, K. Refson and M. C. Payne, Z. Kristallogr. Cryst. Mater., 2005, 220, 567.

9 C. J. Pickard and F. Mauri, Phys. Rev. B, 2001, 63, 245101.

10 M. Profeta, F. Mauri and C. J. Pickard, J. Am. Chem. Soc., 2003, 125, 541-548.

11 S. I. Zones and A. W. Burton, J. Mater. Chem., 2005, 15, 42154223.

12 M. A. Camblor, L. A. Villaescusa and M. J. Díaz-Cabañas, Top. Catal., 1999, 9, 59-76.

13 S.-H. Lee, C.-H. Shin, D.-K. Yang, S.-D. Ahn, I.-S. Nam and S. B. Hong, Microporous Mesoporous Mater., 2004, 68, 97-104.

14 A. Rojas, L. Gomez-Hortiguela and M. A. Camblor, J. Am. Chem. Soc., 2012, 134, 3845-3856.

15 C. M. Zicovich-Wilson, F. Gándara, A. Monge and M. A. Camblor, J. Am. Chem. Soc., 2010, 132, 3461-3471.

16 A. Rojas, M. L. San-Roman, C. M. Zicovich-Wilson and M. A. Camblor, Chem. Mater., 2013, 25, 729-738.

17 T. Blasco, A. Corma, M. J. Díaz-Cabañas, F. Rey, J. A. VidalMoya and C. M. Zicovich-Wilson, J. Phys. Chem. B, 2002, 106, 2634-2642.

18 J. E. Schmidt, D. Xie, T. Rea and M. E. Davis, Chem. Sci., 2015, 6, 1728-1734.

19 J. E. Schmidt, C.-Y. Chen, S. K. Brand, S. I. Zones and M. E. Davis, Chem.-Eur. J., 2016, 22, 4022-4029.

20 L. A. Villaescusa and M. A. Camblor, Chem. Mater., 2016, 28, 7544-7550.

21 Y. Wang, J. Song and H. Gies, Solid State Sci., 2003, 5, 14211433.

22 A. Pulido, G. Sastre and A. Corma, ChemPhysChem, 2006, 7, 1092-1099.

23 A. C. Larson, GSAS: Generalized Structure Analysis System.

24 B. Toby, J. Appl. Crystallogr., 2001, 34, 210-213.

25 J. E. Schmidt, M. W. Deem and M. E. Davis, Angew. Chem., Int. Ed., 2014, 53, 8372-8374. 